\title{
Seizure as presentation of pituitary Adenoma: Brief review of management
}

\author{
Satyarthee GD ${ }^{1 *}$ and Soni $\mathrm{KD}^{2}$ \\ ${ }^{1}$ Department of Neurosurgery, Room no. 714, Neurosciences Centre, AIIMS New Delhi, India \\ ${ }^{2}$ Department of Anaesthesia and critical care, Jai Prakash Narayan Apex trauma centre, AIIMS, New Delhi, India
}

\begin{abstract}
Seizure as initial manifestation of pituitary adenoma is extremely uncommon occurrence. These patients may present with generalized tonic-colonic seizure or rarely focal seizure. A cranial magnetic resonance imaging study involving sellar supra-sellar lesion for search of pituitary adenoma should be advised and carefully examined as these cases represents potential curable patient with surgical resection of pituitary adenoma. Usually Following surgery, shows complete freedom of seizure
\end{abstract}

\section{Introduction}

Pituitary adenoma presenting with seizure as main symptom is rare. However, [1] seizure has been occasionally reported following the pituitary apoplexy [2] or with invasive pituitary adenoma [3]. We report cases of pituitary adenoma who presented with seizure.

\section{Case illustration}

A 35 years old male reported to neurological services with history of recurrent episodes of generalized tonic-clonic seizures for last few years. He was kept on thyroxin replacement therapy along with antiepileptic mediation. General examination revealed feature of hypothyroidism. On neurological examination, visual acuity on both eyes was $6 / 6$. Fundi revealed bilateral pale disc. Rest of the neurological examination was unremarkable. The routine biochemistry and biochemistry profiles were within normal limit. A cranial CT scan showed pituitary macroadenoma. He underwent transsphenoidal total excision of pituitary macroadenoma. Histology revealed evidence of chromophobe pituitary adenoma. He had no further seizure in the post-operative period.

\section{Discussion}

Pituitary adenomas can rarely present with seizures. [1-3] Lundberg et al analyzed 11 patients suffering with invasive pituitary adenoma, two patients suffered from epilepsy, probably related to damage to the temporal lobe cortex.

A newly identified seizure disorder, particularly in adult, invariable raises concern about a brain tumor [4]. About 30\% patients with brain tumors have acute symptomatic seizures as initial symptom or develop in postoperative period. A much smaller proportion of patients have subsequent unprovoked seizure. It is difficult to categorize cases of long-standing epileptics with cerebral neoplasm.

The incidence of seizures among the patients with brain tumors undergoing intracranial surgery is about $25-28 \%$ [4,5] Hauser et al. [6] reported more than $50 \%$ of cases assigned to the idiopathic for all and only $45 \%$ case in the oldest age. Among the young age population (15-35 years) with an identified aetiology CNS infection, CNS tumours, birth defect, and brain trauma were identified as case with equal frequency. In the adult population between age 35 to 64 years trauma and neoplasm were equally responsible, but less frequent than cerebrovascular disease. In contrast, in the elderly population, the most frequent cause was cerebrovascular disease which account for $28 \%$ of all new cases.

Brain tumors accounts for $3.6 \%$ of all cases of epilepsy and $12 \%$ of acquired epilepsy [7]. Epilepsy due to cerebral neoplasm occurs at all ages but is proportionately greater in 25 to 64 years age group and tumors are responsible for a substantial proportion of epilepsy in the elderly [8]. Vakharia et al. [3] reported a 40-year-old harboring trilobed nonfunctional giant pituitary adenoma and observed unique diagnostic and therapeutic challenge and could be managed with aggressive surgical intervention or pharmacologic treatment. $\mathrm{He}$ presented with new onset seizures and cranial magnetic imaging study revealed presence of a trilobed giant pituitary adenoma extending into the frontal lobe anteriorly, posterior to the hypothalamus, and inferior into sella and managed surgically with right pterional craniotomy and excision of tumor. He also noted these large mass lesions should be differentiated from craniopharyngioma, pituitary metastasis, pituitary carcinoma, and a meningioma.

Character of seizure may be able to predict etiological factors [9] Tandon et al. [10] reported late onset seizures, those which changes in character over a period of time, show poor response to antiepileptic medications, prolonged pos-ictal paralysis or present as a status at onset should arouse the suspicion of a neoplastic lesions as causative factor for

${ }^{\star}$ Correspondence to: Guru Dutta Satyarthee MCh, Associate Professor, Department of Neurosurgery, Room no. 714, Neurosciences Centre, AIIMS New Delhi, India, E-mail: duttaguru2002@yahoo.com

Key words: seizure, pituitary adenoma, association, management, surgery, outcome

Received: December 14, 2018; Accepted: December 21, 2018; Published: December 29, 2018 
epilepsy. Commonly, intracerebral pathology is implicated for genesis of seizure. Primary intracerebral tumors presenting with epilepsy are relatively benign in nature [9]. However, extra cerebral pathology i.e. meningioma, which may show their presence by manifestation of seizure.

\section{Conclusion}

Every case presenting with epilepsy should be evaluated in detail with imaging, especially to look for rare causes of extra-cerebral pathology. However, such imaging may require special view for diagnosis and may lead eventually further cost to the patients. But such expense is worthwhile for diagnosis of pathology at early stage with good preservation of the neurological status.

\section{References}

1. Lundberg PO, Drettner B, Hemmingsson A, Stenkvist B, Wide L (1977) The invasive pituitary adenoma. A prolactin-producing tumor. Arch Neurol 34: 742-749. [Crossref]

2. Craig JJ, Gibson JM (2000) Non-convulsive status epilepticus: a treatable cause of confusion in pituitary apoplexy. Br J Neurosurg 14: 141-143. [Crossref]
3. Vakharia RM, Kremen R, Vakharia A, Adarkwah O, Anderson G (2016) Anterior and posterior suprasellar extensions of a symmetrical trilobed nonfunctional giant pituitary adenoma in the sagittal plane: a case report and review of literature. Radiol Case Rep 11:419-424.

4. For PM Copeland GP, Show MDM (1981) The natural history of post-operative Seizures. Acta Neurochair (Wien) 57:15-12

5. Franceschetti S, Battagh AG, Lodrini S. et al. (1988) Relationship between tumors and epilepsy. In: The relational basis of the surgical treatment of epilepsies Broggi G (ed). London: John (ibbey) 1988.

6. Hauser WA, Annegers JF, Kurland LT (1993) Incidence of epilepsy and unprovoked seizures in Rochester, Minnesota: 1935-1984. Epilepsia 34: 453-468. [Crossref]

7. Lühdorf K, Jensen LK, Plesner AM (1986) Etiology of seizures in the elderly. Epilepsia 27: 458-463. [Crossref]

8. Dam AM, Fuglsang-Frederiksen A, Svarre-Olsen U, Dam M (1985) Late-onset epilepsy: etiologies, types of seizure, and value of clinical investigation, EEG, and computerized tomography scan. Epilepsia 26: 227-231. [Crossref]

9. Suri A, Mahapatra AK, Bithal P (1998) Seizures following posterior fossa surgery. $\mathrm{Br} J$ Neurosurg 12: 41-44. [Crossref]

10. Tandon PN, Mahapatra AK, Khosla A (2001) Epileptic seizures in supratentorial gliomas. Neurol India 49: 55-59. [Crossref]

Copyright: $(2018$ Satyarthee GD. This is an open-access article distributed under the terms of the Creative Commons Attribution License, which permits unrestricted use, distribution, and reproduction in any medium, provided the original author and source are credited. 